\title{
SKEW GROUP RINGS AND MAXIMAL ORDERS
}

\author{
by R. MARTIN
}

(Received 18 January, 1994; revised 31 May, 1994)

Introduction. Let $S$ be a prime Noetherian ring and $G$ a finite group acting on $S$ such that $G$ is $X$-outer on $S$. We give sufficient conditions for the skew group ring $S * G$ to be a prime maximal order. If we impose the further hypothesis that the order of $G$ be a unit of $S$, then these conditions are also necessary. Moreover, if $S$ is a commutative Noetherian domain, then there are necessary and sufficient conditions for $S * G$ to be a prime maximal order, without requiring that the order of $G$ be a unit in $S$.

1.1 Given a ring $S$ and a group $G$ acting on $S$ we write $s^{g}$ for the image of $s \in S$ under the action of $g \in G$, and define the skew group ring $S * G$ to be the ring which is a free left $S$-module with the elements of $G$ as a basis. Multiplication is extended linearly from $S$ and $G$, and $g s=s^{g} g$ for all $s \in S, g \in G$. Our aim is to prove the following result.

THEOREM 3.13. (Main Theorem). Let $S$ be a prime Noetherian ring and $G$ a finite group acting on $S$ such that $G$ is $X$-outer. Let $T=S * G$ be the skew group ring, and denote by $\Omega_{0}$ the set of reflexive height-1 G-prime ideals of $S$. Consider the following hypotheses:

(a) $S$ is a $G$-maximal order;

(b) $p_{0} T$ is a prime ideal of $T$ for all $p_{0} \in \Omega_{0}$.

(i) If (a) and (b) both hold, then $T$ is a prime maximal order.

(ii) Suppose that the order of $G$ is a unit of $S$. If $T$ is a (prime) maximal order, then (a) and (b) both hold.

Here, a $G$-maximal order is an order which is not properly contained in any $G$-invariant order to which it is equivalent; see 2.1 for the precise definition.

We give an example (Example 3.14) to show that statement (ii) of the Main Theorem does not hold without the additional hypothesis that the order of $G$ be a unit in $S$.

Compare our results with [12, Theorem 3.1], where sufficient conditions are given for a ring $R$, strongly graded by a finite group $G$ with the order of $G$ a unit in $R$, to be a tame order. A tame order is a generalisation of the concept of a maximal order; a prime Noetherian maximal order integral over its centre is a tame order.

We also prove a result, namely Theorem 4.6, that is the commutative analogue of Theorem 3.13; as we shall see, no hypothesis on the order of $G$ is required in this case.

1.2 See [13, Section 12] for a definition of $X$-outer. Let $R$ be a ring and $G$ a group. In Section 2 we offer some results concerning $G$-maximal orders. As is to be expected, many of these are analogues of results in the (ordinary) maximal order case. In Section 3 we turn to the proof of the Main Theorem, a key tool of which is Theorem 3.2 (which we refer to as the Test Theorem); we also give a $G$-equivariant version of this as Theorem 3.3. The Test Theorem is partially proved in [1, Proposition $1.10(\mathrm{~b})]$ and $[7$, Lemma 1.2(ii)], and, although the result is undoubtedly well-known, a suitable reference for the complete proof could not be found. Section 4 is concerned with the proof of the analogue of the Main Theorem for the case where the coefficient ring of $T$ is a commutative

Glasgow Math. J. 37 (1995) 249-263. 
Noetherian domain. As we shall see, we do not require the hypothesis that the order of $G$ be a unit in $S$.

1.3 A full account of maximal orders can be found in [8] and [10]. Our main source for skew group rings was [13]. Throughout, the Jacobson radical and quotient ring of any ring $A$ will be denoted by $J(A)$ and $Q(A)$ respectively. The results of this paper form part of the author's Ph.D. thesis at the University of Glasgow, under the supervision of Professor K. Brown. I would like to thank Professor H. Marubayashi (Naruto) for his careful reading of an earlier version of this paper.

\section{2. $G$-Maximal orders.}

2.1 Definitions. Let $R$ be a prime Noetherian ring with simple Artinian quotient ring $Q$. Then $R$ is an order in $Q$ and an order $S$ in $Q$ is said to be equivalent to $R$ if there exist units $a, b, c, d$ in $Q$ such that $a R b \subseteq S$ and $c S d \subseteq R$. We call $R$ a maximal order if it is maximal within its equivalence class; that is if $S$ is an order in $Q$ equivalent to $R$ and containing $R$, then $S$ must be equal to $R$. Let $I$ be a non-zero ideal of $R$. Define $\mathrm{O}_{l}(I)=\{q \in Q: q I \subseteq I\}$ and $\mathrm{O}_{r}(I)=\{q \in Q: I q \subseteq I\}$. Then $\mathrm{O}_{l}(I)$ and $\mathrm{O}_{r}(I)$ are orders in $Q$ equivalent to $R$. The following lemma is an easy consequence of [10, Proposition 1.3.1].

Lemma. Let $R$ be a prime Noetherian ring with the property that $\mathrm{O}_{l}(P)=\mathrm{O}_{r}(P)=R$, for all (non-zero) prime ideals $P$ of $R$. Then $R$ is a maximal order.

Let $R$ be a prime Noetherian ring and $G$ a finite group acting on $R$ with $G \subseteq \operatorname{Aut}(R)$. It follows that $G$ acts on the quotient ring $Q$ of $R$; in particular, $G$ permutes the set of right orders in $Q$. Let $S$ be a $G$-invariant right order in $Q$. We say that $R$ is equivalent to $S$ (written $R \sim S$ ) if there exist units $a, b, c, d$ in $Q$ such that $a R b \subseteq S$ and $c S d \subseteq R$. It is easy to check that $\sim$ is an equivalence relation.

The ring $R$ is said to be a $G$-maximal right order in $Q$ if $R$ is strictly contained in no other $G$-invariant right order to which it is equivalent. $G$-maximal left orders and $G$-maximal orders are defined similarly. (Compare this definition to that of a maximal order given above.)

Suppose that $I$ is a proper $G$-invariant ideal of a $\operatorname{ring} R$ on which a group $G$ acts. We say that $I$ is a $G$-prime ideal of $R$ if, for all $G$-invariant ideals $A, B$ of $R$, the inclusion $A B \subseteq R$ forces either $A \subseteq I$ or $B \subseteq I$.

We have the following analogue of Lemma 2.1 that is proved in a similar way.

2.2 Proposition. Let $R$ and $G$ be as in 2.1. Then $R$ is a G-maximal order if and only if $\mathrm{O}_{l}(P)=\mathrm{O}_{r}(P)=R$, for all non-zero G-prime ideals of $P$ of $R$.

The next result shows that the concept of a $G$-maximal order yields nothing new in the commutative case.

2.3 Lemma. Let $S$ be a commutative Noetherian domain and $G$ a finite group acting as automorphisms on $S$. Then $S$ is a G-maximal order if and only if $S$ is a maximal order.

Proof. It is clear from the definitions that if $S$ is a maximal order then $S$ is a $G$-maximal order.

Conversely, suppose that $S$ is a $G$-maximal order which is not a maximal order. By [8, Lemma 5.3.2] it is enough to show that, if $S$ is contained in a subring $T$ of $Q=Q(S)$ with $T=\sum\left\{y_{i} S: i=1, \ldots, n\right\}$ a finitely generated $S$-module, then $T=S$. Given such a 
ring $T$, put $\hat{T}:=\left\langle T^{g}: g \in G\right\rangle$. Then $\hat{T}$ is a $G$-invariant subring of $Q$ containing $S$ and $T$. Also $\hat{T}$ is a finitely generated $S$-module, for, consider any monomial

$$
\hat{y}=y_{i_{1}}^{g_{1}} y_{i_{2}}^{g_{2}} \ldots y_{i_{t}}^{g_{t}}
$$

in the $G$-conjugates of $\left\{y_{1}, \ldots, y_{n}\right\}$ with $t>|G|$. It is clear that $\hat{y}$ involves components of the form (say) $y_{j}^{g}$ and $y \xi$, for some $g \in G$. Since $Q$ is commutative, we can write $\hat{g}$ (with rearrangement if necessary) so that $y_{j}^{g}, y_{k}^{g}$ are adjacent. Then we can express $y_{j}^{g} y_{k}^{g}$ as an $S$-linear combination of $\left\{y_{1}^{g}, \ldots, y_{n}^{g}\right\}$. Hence $\hat{T}$ is a finitely generated $S$-module and so, since $S$ is a $G$-maximal order by hypothesis, $\hat{T}=S$. Hence $T=S$ and $S$ is a maximal order, as required.

2.4 Example. We now give an example to show that (non-commutative) $G$-maximal orders which are not maximal orders do exist. Let $R=\mathbb{Z}_{2 \mathbb{Z}}$ and put

$$
S=\left(\begin{array}{cc}
R & R \\
2 R & R
\end{array}\right)
$$

a prime Noetherian ring. Let

$$
g=\left(\begin{array}{cc}
0 & \sqrt{\frac{1}{2}} \\
\sqrt{2} & 0
\end{array}\right) \in M_{2}(\mathbb{R})
$$

Put $G:=\langle g\rangle \cong C_{2}$, the cyclic group of order 2 . It is easy to check that $G$ acts on $S$ by conjugation, viewing $S$ as a subring of $M_{2}(\mathbb{R})$, with

$$
\left(\begin{array}{cc}
a & b \\
2 c & d
\end{array}\right)^{g}=\left(\begin{array}{cc}
d & c \\
2 b & a
\end{array}\right)
$$

for $a, b, c, d \in \mathbb{Z}$. Consider the height-1 prime ideal

$$
P=\left(\begin{array}{ll}
2 R & R \\
2 R & R
\end{array}\right)
$$

of $S$. Then

$$
P^{g}=\left(\begin{array}{cc}
R & R \\
2 R & 2 R
\end{array}\right)
$$

$P$ and $P^{g}$ are the only non-zero prime ideals of $S$, and both have height one. One can check that

$$
x=\left(\begin{array}{ll}
0 & 0 \\
1 & 0
\end{array}\right) \in \mathrm{O}_{l}(P) \backslash S
$$

and so $S$ is not a maximal order. However, $S$ is a $G$-maximal order: by Proposition 2.2 it is enough to show that

$$
\mathrm{O}_{l}\left(P \cap P^{g}\right)=\mathrm{O}_{r}\left(P \cap P^{g}\right)=S
$$


To this end, let $\alpha \in \mathrm{O}_{l}\left(P \cap P^{g}\right)$ and put

$$
\alpha=\left(\begin{array}{ll}
x & y \\
z & w
\end{array}\right)
$$

where $x, y, z, w \in Q(S)$, the quotient ring of $S$. Let

$$
\left(\begin{array}{cc}
2 a & b \\
2 c & 2 d
\end{array}\right)
$$

be a non-zero element of $P \cap P^{g}$. Then

$$
\left(\begin{array}{ll}
x & y \\
z & w
\end{array}\right)\left(\begin{array}{cc}
2 a & b \\
2 c & 2 d
\end{array}\right)=\left(\begin{array}{cc}
2(a x+c y) & b x+2 d y \\
2(a z+c w) & b z+2 d w
\end{array}\right) \in\left(\begin{array}{cc}
2 R & R \\
2 R & 2 R
\end{array}\right)=P \cap P^{g}
$$

Since $a, b, c, d$ can be arbitrary integers, we must have that $x, y, w \in R$ and $z \in 2 R$ so that $\alpha \in S$. Hence $\mathrm{O}_{l}\left(P \cap P^{g}\right)=S$. Similarly, $\mathrm{O}_{r}\left(P \cap P^{g}\right)=S$ and $S$ is a $G$-maximal order, as claimed.

The following result is a $G$-equivariant version of [7, Lemma 2.1 (ii)], and since the proof of our result is similar to the proof of theirs, we do not include it here.

2.5 Proposition. Let $R$ be a prime Noetherian $G$-maximal order, where $G$ is a finite group acting on $R$. If $P$ is a reflexive height-1 $G$-prime ideal of $R$, then $P$ is localisable.

2.6 Definimion. For a non-zero ideal $I$ of a prime Noetherian ring $R$, put $I_{l}^{*}=\{q \in Q(R): q I \subseteq R\}$ and $I_{r}^{*}=\{q \in Q(R): I q \subseteq R\}$. When $R$ is a maximal order, we have $I_{l}^{*}=I_{r}^{*}:=I^{*}$ by $[8$, Proposition 5.1.8]. The ideal $I$ is said to be invertible if $\left(I_{l}^{*}\right) I=I\left(i_{r}^{*}\right)=R$, and $I$ is reflexive when $\left(I_{l}^{*}\right)_{r}^{*}=\left(i_{r}^{*}\right)_{1}^{*}=I$.

By a local ring $R$, we mean that $R / J(R)$ is simple Artinian; $R$ is semilocal if $R / J(R)$ is semisimple Artinian.

2.7 Proposition. Let $R$ be a prime Noetherian local ring with $J:=J(R)$ reflexive. Then the following are equivalent:

(i) $R$ is a maximal order;

(ii) $R$ is hereditary;

(iii) $J$ is invertible.

Proof. (ii) $\Leftrightarrow$ (iii). [6, Proposition 1.3].

(i) $\Rightarrow$ (iii). It is clear that $J^{*} J$ is a non-zero ideal of $R$. Since $\mathrm{O}_{l}(J)=R, J \subset J^{*} J$. Due to the maximality of $J$ we must have $J^{*} J=R$. Similarly, $J J^{*}=R$, and so $J$ is invertible.

(iii) $\Rightarrow$ (i). Suppose that $J$ is invertible, but that $R$ is not a maximal order. Let $0 \neq I$ be an ideal of $R$ such that $R \subset \mathrm{O}_{l}(I)$, and choose $I$ to be maximal with respect to this property. Now, $\mathrm{O}_{l}(J) J \subseteq J$ implies that

$$
\mathrm{O}_{l}(J)=\mathrm{O}_{l}(J) J\left(J_{r}^{*}\right) \subseteq J\left(J_{r}^{*}\right)=R
$$

since $J$ is invertible. Hence $\mathrm{O}_{l}(J)=R$. A similar argument gives $\mathrm{O}_{r}(J)=R$. Therefore by choice of $I, I \subset J$ and it follows that $J_{r}^{*}=J_{l}^{*}=J^{*}$. Put $K=I J^{*}$, an ideal of $R$. Then 
$K J=I J^{*} J=I$, since $J$ is invertible. It is clear that $I \subseteq K$; suppose that $I=K$. Then $K J=K$, contradicting Nakayama's Lemma. Therefore $I \subset K$.

Let $x \in \mathrm{O}_{l}(I)$. Then $x I \subseteq I$ so that $x K J \subseteq K J$. We have

$$
x K=x K J J^{*} \subseteq K J J^{*}=K
$$

since $J$ is invertible, and so $x \in \mathrm{O}_{l}(K)$. Therefore $R \subset \mathrm{O}_{l}(I) \subseteq \mathrm{O}_{l}(K)$ with $I \subset K$, contradicting the initial choice of $I$. Hence there exists no such ideal $I$. A similar argument works on the right, and so $R$ is a maximal order. This completes the proof.

The next result is a generalisation of (i) $\Rightarrow$ (iii) of Proposition 2.7. Here, and in 2.9, we write $M_{0}$ for $\bigcap\left\{M^{g} ; g \in G\right\}$, where $M$ is an ideal of a ring $R$ on which a group $G$ acts.

2.8 Proposmion. Let $R$ be a prime Noetherian semilocal ring and $G$ a finite group acting on $R$. Suppose that $J:=J(R)$ is reflexive. If $R$ is a $G$-maximal order, then $J$ is invertible.

Proof. We claim that each $M_{0}$ is reflexive. Fix a maximal ideal $M$ of $R$. Since $R$ is a $G$-maximal order, it is enough to show that $M_{0}$ is the right annihilator of a non-zero submodule of $Q / R$ (where $Q$ is the quotient ring of $R$ ). Now, since $J$ is right reflexive by hypothesis, there exists a submodule $A$ of $Q$ strictly containing $R$ with $J=\operatorname{rann}(A / R)$. Since $R$ is semilocal, $R / J$ is semisimple Artinian. Therefore $R / J$ is isomorphic to a direct sum of matrix rings over division rings, say $R / J \cong \bigoplus_{i=1}^{t} M_{n_{i}}\left(D_{i}\right)$, where $n_{i}$ is a natural number and each $D_{i}$ is a division ring. We can also write $M_{0} / J \cong \bigoplus_{i=1}^{r} M_{n_{i}}\left(D_{i}\right)$, with reindexing if necessary, for some $r<t$. Since $A / R$ is a faithful $R / J$-module, $A / R$ includes at least one copy of each isomorphism class of irreducible $R / J$-modules in its decomposition. For each $i=1, \ldots, t$ let $V_{i}$ be an irreducible $M_{n_{i}}\left(D_{i}\right)$-module. Let $B / R$ denote the sum in $A / R$ of all the irreducible $R / J$-modules isomorphic to $V_{i}$, where $i=r+1, \ldots, t$. It follows that $M_{0}$ is the right annihilator (in $R$ ) of $B / R$. Therefore $M_{0}$ is right reflexive.

We now show that $M_{0}$ is invertible. Now, $M_{0}^{*} M_{0}$ is an ideal of $R$ containing $M_{0}$, and is easily checked to be $G$-invariant. If $M_{0}^{*} M_{0} \subset R$, then $M_{0}^{*} M_{0} \subseteq M^{g}$, for some $g \in G$. Then

$$
M_{0}^{*} M_{0}=\bigcap\left\{\left(M_{0}^{*} M_{0}\right)^{g}: g \in G\right\} \subseteq \bigcap\left\{M^{g}: g \in G\right\}=M_{0},
$$

so that $M_{0}^{*} \subseteq \mathrm{O}_{l}\left(M_{0}\right)=R$, since $R$ is a $G$-maximal order. This is a contradiction to the reflexivity of $M_{0}$, and so $M_{0}^{*} M_{0}=R$. Similarly $M_{0} M_{0}^{*}=R$ and $M_{0}$ is indeed invertible. The ideals $M_{0}$ are maximal $G$-invariant, so one sees easily, adapting the proof of [8, Theorem 5.2.10], that $\bigcap\left\{M_{0}: M\right.$ maximal $\}=\Pi\left\{M_{0}: M\right.$ maximal $\}$. Since a product of invertible ideals is again invertible, the result follows.

2.9 Corollary to Proposition 2.8. Let $R$ be a prime Noetherian semilocal ring and $G$ a finite group acting on $R$. Suppose that $J:=J(R)$ is reflexive. Then the following are 
equivalent:

(i) $R$ is a G-maximal order;

(ii) $M_{0}$ is invertible for each maximal ideal $M$ of $R$.

Proof. (i) $\Rightarrow$ (ii). This follows from the proof of Proposition 2.8.

(ii) $\Rightarrow$ (i). Suppose that $M_{0}$ is invertible for each maximal ideal $M$ of $R$. Then $J$ is also invertible. Suppose that $R$ is not a $G$-maximal order, so that there exists a non-zero $G$-invariant ideal $I$ of $R$ such that $R \subset \mathrm{O}_{l}(I)$. Choose $I$ to be maximal with respect to this property. Now, since $I$ is $G$-invariant, $I$ is contained in $M_{0}$ for some maximal ideal $M$ of $R$. Since $M_{0}$ is invertible, $M_{0}$ is (right and left) projective by [8, Lemma 5.2.5]. It now follows from [8, Section 5.1.7] that $M_{0}$ is reflexive.

Put $K=I M_{0}^{*}$, a $G$-invariant ideal of $R$. We have $I=I M_{0}^{*} M_{0}=K M_{0} \subseteq K$; suppose that $I=K$. Then $K M_{0}=K$, so that $K=K M_{0}=\left(K M_{0}\right) M_{0}=\ldots=K\left(M_{0}\right)^{t}$ for all $t \geqslant 0$. Therefore $K \subseteq \bigcap\left\{\left(M_{0}\right)^{i}: i=1, \ldots, \infty\right\}$. But $M_{0}$ being invertible means that $M_{0}$ has the $A R$-property, by [8, Corollary 4.2.5], and so $\bigcap\left\{\left(M_{0}\right)^{i}: i=1, \ldots, \infty\right\}=0$ by [6, Corollary 2.2]. Hence $K=0$, a contradiction, and so $I \subset K$.

In the same way, as was shown in the proof of Proposition 2.7, we have $R \subset \mathrm{O}_{l}(I) \subseteq \mathrm{O}_{l}(K)$. This contradicts our initial choice of $I$, and so $R$ is a $G$-maximal order. Thus (i) holds and the proof is complete.

\section{The Main Theorem.}

3.1 Let $R$ be a prime Noetherian ring with quotient ring $Q$, and recall from 2.6 the definitions of $I_{l}^{*}$ and $I_{r}^{*}$ for an ideal $I$ of $R$. Define

$$
\begin{aligned}
& R_{0}:=\{q \in Q: q I \subseteq R \text { for some non-zero ideal } I \text { of } R\}, \\
& R_{0}^{\prime}:=\{q \in Q: I q \subseteq R \text { for some non-zero ideal } I \text { of } R\} .
\end{aligned}
$$

Note that

$$
R_{0}=\bigcup\left\{I_{l}^{*}: 0 \neq I \text { an ideal of } R\right\} \text { and } R_{0}^{\prime}=\bigcup\left\{I_{r}^{*}: 0 \neq I \text { an ideal of } R\right\} .
$$

Suppose that $R$ is a maximal order. Then, since $I_{l}^{*}=I_{r}^{*}$ for each $I$ of $R$, we see that $R_{0}=R_{0}^{\prime}$.

In the proof of the main result of this paper, we will use the following theorem (referred to as the Test Theorem). This result-which is probably well-known-is partially proved in the literature; in particular, that $R$ is a maximal order implies (iv) is shown in [1, Proposition 1.10(b)], and that $R$ is a maximal order implies (i) is given in [7, Lemma 1.2(ii)]. For further details of the complete proofs of Theorem 3.2 and Theorem 3.3 , see [9].

3.2 THEOREM. (Test Theorem). Let $R$ be a prime Noetherian ring and denote by $\Omega$ the set of all height-1 reflexive prime ideals of $R$. Then $R$ is a maximal order if and only if the following conditions hold:

(i) each $P \in \Omega$ is localisable;

(ii) $R_{P}$ is a maximal order for all $P \in \Omega$;

(iii) $R_{0}=R_{0}^{\prime}$;

(iv) $R=R_{0} \cap\left(\cap\left\{R_{P}: P \in \Omega\right\}\right)$. 
We also have a $G$-equivariant version of the Test Theorem. It is proved by making straightforward adjustments to the proof of Theorem 3.2, using, for example, Proposition 2.5 to deduce (i).

3.3 THEOREM. Let $S$ be a prime Noetherian ring and $G$ a finite group acting on $S$. Denote by $\Gamma$ the set of all height -1 reflexive $G$-prime ideals of $S$. Then $S$ is a $G$-maximal order if and only if the following conditions hold:

(i) $p_{0}$ is localisable, for all $p_{0} \in \Gamma$;

(ii) $S_{p_{0}}$ is a $G$-maximal order, for all $p_{0} \in \Gamma$;

(iii) $S_{0}=S_{0}^{\prime}$ :

(iv) $S=S_{0} \cap\left(\cap\left\{S_{p_{0}}: p_{0} \in \Gamma\right\}\right)$.

3.4 Notation AND Hypotheses. Unless stated otherwise, the following will apply. Let $S$ be a prime Noetherian ring and $G$ a finite group acting on $S$. Suppose that the action of $G$ is $X$-outer on $S$. Denote the skew group ring $S * G$ by $T$. Let

$$
\begin{aligned}
& \Omega=\{p \in \operatorname{Spec}(S): p \text { is reflexive and has height } 1\}, \\
& \Omega_{0}=\left\{p_{0}: p_{0}=\bigcap\left\{p^{g}: g \in G\right\}, \text { for some } p \in \Omega\right\} .
\end{aligned}
$$

Note that, under the above hypotheses, [13, Corollary 12.6] guarantees that $T$ is prime.

3.5 Lemma. Let $S, G$ and $T$ be as in 3.4. Then $T_{0}=S_{0} * G$.

Proof. It is easy to see that $T$ has an Artinian quotient ring $Q(T)$, and that $Q(T)=Q(S) * G$. Let $x \in T_{0} \subseteq Q(T)=Q(S) * G$, and write $x=\sum\left\{s_{g} g: g \in G\right\}$ with $s_{g} \in$ $Q(S)$ for each $g \in G$. By the definition of $T_{0}$, there exists a non-zero ideal $l$ of $T$ with $x I \subseteq T$. Now $T$ is prime so that, by Goldie's Theorem, $Q(T)$ is simple Artinian. Therefore

$$
Q(T)=I Q(T)=I(Q(S) * G)=I G(Q(S))=I(Q(S)) .
$$

Hence there exist elements $a \in I$ and $c \in C_{S}(0)$ with $1=a c^{-1}$, and so $a=c \in I \cap S$. It follows that $I \cap S$ is a non-zero $G$-invariant ideal of $S$. Therefore $(I \cap S) * G$ is a non-zero ideal of $T$ contained in $I$. We have $x((I \cap S) * G) \subseteq x I \subseteq T$, so that

$$
\left(\sum_{g \in G} s_{g} g\right)((I \cap S) * G) \subseteq T=S * G .
$$

This means that $s_{g}(I \cap S) \subseteq S$, for all $g \in G$, since $I \cap S$ is $G$-invariant. Therefore $s_{g} \in S_{0}$ for all $g \in G$ and so $x \in S_{0} * G$. The reverse inclusion is similar, but easier.

3.6 Remark. It is easily checked that an ideal $p_{0}$ of $S$ belongs to $\Omega_{0}$ if and only if it is $G$-prime, reflexive and has height 1 . In particular, if $S$ is a $G$-maximal order, $p_{0}$ is localisable, by Proposition 2.5. In fact, $C_{S}\left(p_{0}\right)$ is an Ore set in $T$, by an argument similar to the proof of [16, Lemma 2.6]. Equivalently, the ideal $\sqrt{\left(p_{0} T\right)}$ of $T$ is a localisable semiprime ideal of $T$. We denote the localisation of $T$ at $\sqrt{\left(p_{0} T\right)}$ by $T_{p_{0}}$; note that by construction, $T_{p_{0}}=S_{p_{0}} * G$.

3.7 Lemma. Adopt the notation of 3.4 and suppose that $S$ is a $G$-maximal order. then $T=T_{0} \cap\left(\bigcap_{p_{0} \in \Omega_{0}} T_{p_{0}}\right)$. 
Proof. If $S$ is a $G$-maximal order, then by Theorem 3.3,

Therefore

$$
S=S_{0} \cap\left(\bigcap_{p_{0} \in \Omega_{0}} S_{p_{0}}\right)
$$

It now follows that

$$
\begin{aligned}
T=S * G & =\left(S_{0} \cap\left(\bigcap_{p_{0} \in \Omega_{0}} S_{p_{0}}\right)\right) * G \\
& =\left(S_{0} * G\right) \cap\left(\bigcap_{p_{0} \in \Omega_{0}}\left(S_{p_{0}} * G\right)\right) .
\end{aligned}
$$

$$
T=T_{0} \cap\left(\bigcap_{p_{0} \in \Omega_{0}} T_{p_{0}}\right)
$$

by Lemma 3.5 and Remark 3.6, as required.

3.8 Lemma. Adopt the notation of 3.4 and suppose that $T=S * G$ is a (prime Noetherian) maximal order. Then $S$ is a G-maximal order.

Proof. Let $I$ be a non-zero $G$-invariant ideal of $S$. Then $\hat{I}:=I * G$ is a non-zero ideal of $T$. Now, $\mathrm{O}_{l}(I) I \subseteq I$ implies that

$$
O_{l}(I) \hat{I}=O_{l}(I)(I * G) \subseteq I * G=\hat{I} .
$$

Therefore $\mathrm{O}_{l}(I) \subseteq \mathrm{O}_{l}(\hat{I})=T$, since $T$ is a maximal order. Let $Q$ denote the quotient ring of $S$. We have $\mathrm{O}_{l}(I) \subseteq T \cap Q=S$, so that $\mathrm{O}_{,}(I)=S$. Since $I$ is $G$-invariant $\hat{I}=I * G=$ $G * I$, so that we can use symmetry along with the above argument to show that $\mathrm{O}_{r}(I)=S$. Hence by Proposition $2.2 S$ is a $G$-maximal order, as required.

3.9 Lemma. Adopt the notation and hypotheses of 3.4. Suppose further that $S$ is a semilocal G-maximal order with $J(S)$ reflexive, and that $|G|^{-1} \in S$. Then $T$ is a semilocal hereditary ring.

Proof. First we show that $T$ is semilocal. Since $|G|^{-1} \in S,[13$, Theorem 4.2] gives that $J(T)=J(S) * G$. Now $S$ is semilocal, and so $S / J(S)$ is semisimple Artinian. We have

$$
T / J(T)=(S * G) /(J(S) * G) \cong(S / J(S)) * G,
$$

an Artinian ring. Hence $T$ is semilocal, as claimed. By Proposition $2.8, J(S)$ is invertible. Therefore

$$
\begin{aligned}
J(T)(J(T))^{*}=(J(S) * G)(J(S) * G)^{*} & =(J(S) * G)\left(J(S)^{*} * G\right) \\
& =J(S)(J(S))^{*} * G=S * G=T .
\end{aligned}
$$

Similarly $(J(T)) * J(T)=T$, and $J(T)$ is also invertible.

Put $J:=J(T)$. By $[8, \text { Lemma 5.2.5] }]_{T} J$ and $J_{T}$ are projective and so pr.dim. $(T / J)_{T}=1$. Therefore by [8, Theorem 7.3.14]

$$
\operatorname{gl} . \operatorname{dim} .(T) \leqslant \operatorname{gl} . \operatorname{dim} .(T / J)+\operatorname{pr} \cdot \operatorname{dim} .(T / J)=0+1=1 .
$$

Hence $T$ is hereditary, as required. 
3.10 Remark. Adopt the notation of 3.4. It follows from easy adaptations of [13, Lemma 14.1] and [2, Theorem 1.6], together with the initial comment of Remark 3.6, that if $P$ is a height- 1 reflexive prime ideal of $T$, then $p_{0}:=P \cap S$ belongs to $\Omega_{0}$. In particular, $p_{0}$ is $G$-prime and so $p_{0} T$ is an ideal of $T$, and is contained in $P$. The following results give sufficient conditions for equality.

3.11 Lemma. Let $S$ be a Noetherian ring and $G$ a finite group acting on $S$. Let $T$ denote the skew group ring $S * G$. Let $P$ be a prime ideal of $T$ and put $p_{0}=P \cap S$. Suppose that $p_{0}$ is localisable and that $P T_{p_{0}}=p_{0} T_{p_{0}}$. Then $P=p_{0} T$.

Proof. As explained in Remark 3.6, $C_{S}\left(p_{0}\right)$ is an Ore set in $T$, and so $T_{p_{0}}$ exists. It is clear that $p_{0} T \subseteq P$. For the reverse inclusion, let $\alpha \in P$ and write $\alpha=a c^{-1}$ with $a \in p_{0} T$ and $c \in C_{S}\left(p_{0}\right)$. Then $\alpha c=a \in p_{0} T$. But $c \in C_{S}\left(p_{0}\right) \subseteq C_{T}\left(p_{0} T\right)$, and so $\alpha c \in p_{0} T$ implies that $\alpha \in p_{0} T$ as required.

3.12 Proposirion. Adopt the notation and hypotheses of 3.4 and suppose that $|G|^{-1} \in S$. Suppose further that $T$ is a prime Noetherian maximal order. Let $P$ be a reflexive prime ideal of $T$ and put $p_{0}=P \cap S$. Then $P=p_{0} T$.

Proof. By Lemma 3.8, $S$ is a $G$-maximal order. Then, by Remark 3.10, $p_{0} \in \Omega_{0}$, and so is localisable by Remark 3.6. First we localise $S$ at $p_{0}$, so that without loss of generality, in view of Lemma $3.11, S$ is a semilocal ring. Of course, $G$ is still outer on $S$. Lemma 3.9 now applies to give that $T$ is semilocal and hereditary. By [8, Theorem 5.2.10], we see that each finitely generated projective $T$-module is a generator. In particular, this is so for the projective $T$-module $\hat{G} T$, where $\hat{G}=\sum_{g \in G} g$. Since $\operatorname{End}_{T}(\hat{G} T) \cong S^{G}$ by [8, Proposition 7.8.5], it follows that $T=M_{n}(W)$, where $W \cong S^{G}$ (and in fact $n=|G|$ ).

The result we seek will follow if we can display an isomorphism $\theta$ from $T$ to $M_{n}\left(S^{G}\right)$ for which the inverse image of the scalar matrices is the subring $S^{G}$ of $T$. First, note that $S^{G}$ is a prime hereditary semilocal Noetherian ring by Morita theory. Since $u$ $\operatorname{dim}_{S^{G}}(S)=$ n.u-dim $S^{c}\left(S^{G}\right)$ from the isomorphism of the previous paragraph, [4] ensures that $S$ is a free $S^{G}$-module of rank $n$. Now the Morita context connecting $S^{G}$ with $T[\mathbf{8}$, $\S 7.8]$ is in fact a Morita equivalence, as can be seen either from the isomorphism already mentioned, or from [8, Proposition 3.5.6] and the fact that $T \hat{G} T=T$; the latter holds since $T \hat{G} T$ is an idempotent invertible ideal [8, Proposition 5.2.6 and Theorem 5.2.10].

Thus $T \cong \operatorname{End}_{S^{c}}\left({ }_{s^{c}} S\right)$. Realising this isomorphism via a basis $\left\{1, s_{2}, \ldots, s_{n}\right\}$ of ${ }_{s^{G}} S$ (whose existence follows using the trace map) yields the desired isomorphism $\theta$.

We are now in a position to prove the main result of this section, which is as follows.

3.13 THEOREM. Adopt the notation and hypotheses of 3.4 and consider the following hypotheses:

(a) $S$ is a $G$-maximal order;

(b) $p_{0} T$ is a prime ideal of $T$, for all $p_{0} \in \Omega_{0}$.

(i) If (a) and (b) both hold, then $T$ is a prime maximal order.

(ii) Suppose that the order of $G$ is a unit in $S$. If $T$ is a (prime) maximal order, then (a) and (b) both hold. 
Proof. (i) Suppose first that (a) and (b) both hold. Since $S$ is a $G$-maximal order by (a), any ideal $p_{0}$ in $\Omega_{0}$ is localisable by Proposition 2.5. By Remark 3.6, $C_{S}\left(p_{0}\right)$ is an Ore set in $T$ and $T_{p_{0}}$ exists. Recall that $T$ is prime. Now

$$
T / p_{0} T=(S * G) /\left(p_{0} * G\right) \cong\left(S / p_{0}\right) * G,
$$

for all $p_{0} \in \Omega_{0}$. Therefore $p_{0} T$ prime implies that $\left(S / p_{0}\right) * G$ is prime for all such $p_{0}$. Then $Q\left(S / p_{0}\right) * G=Q\left(\left(S / p_{0}\right) * G\right.$ is simple Artinian. But

$$
Q\left(\frac{S}{p_{0}}\right) * G \cong\left(\frac{S_{p_{0}}}{p_{0} S_{p_{0}}}\right) * G=\frac{\left(S_{p_{0}} * G\right)}{\left(p_{0} S_{p_{0}} * G\right)},
$$

so that $p_{0} S_{p_{0}} * G$ is a prime ideal of $S_{p_{0}} * G$. We have that $J\left(S_{p_{0}}\right) * G$ is prime. Using $[13$, Theorem 4.2] we see that

$$
\left(J\left(T_{p_{0}}\right)\right)^{n} \subseteq J\left(S_{p_{0}}\right) * G \subseteq J\left(T_{p_{0}}\right)
$$

for some $n \in \mathbb{N}$. But since $J\left(S_{p_{0}}\right) * G$ is prime, we must have

$$
J\left(S_{p_{0}}\right) * G=J\left(T_{p_{0}}\right),
$$

so that $J\left(T_{p_{0}}\right)$ is prime. Therefore $\frac{T_{p_{0}}}{J\left(T_{p_{0}}\right)}$ is simple Artinian, and so $T_{p_{0}}$ is local. Now $S$ is a $G$-maximal order, and so by Theorem $3.3, S_{p_{0}}$ is a $G$-maximal order. Therefore $S_{p_{0}}$ is hereditary, by Corollary 2.9 , and so $J\left(S_{p_{0}}\right)$ is projective. Thus, since $T_{p_{0}}$ is a free left $S_{p_{0}}$-module, $J\left(T_{p_{0}}\right)=J\left(S_{p_{0}}\right) \otimes_{S_{p_{0}}} G$ is a projective $T_{p_{0}}$-module, and hence is reflexive. We can now apply Proposition 2.7 to give that $T_{p_{0}}$ is a maximal order.

Observe that, if $P$ is a reflexive height-1 prime of $T$, then $p_{0}:=P \cap S$ belongs to $\Omega_{0}$, by Remark 3.10. Since $P$ is minimal over $p_{0} T$, by [13, Theorem 16.6], hypothesis (b) guarantees that $P=p_{0} T$. By this fact and Lemma 3.7,

$$
T=T_{0} \cap\left(\bigcap_{p_{0} \in \Omega_{0}} T_{p_{0}}\right)=T_{0} \cap\left(\bigcap_{P} T_{P}\right),
$$

where $P$ runs over the set of height- 1 reflexive primes of $T$. Moreover, $T_{0}=T_{0}^{\prime}$, by Lemma 3.5. Now Theorem 3.2 (the Test Theorem) gives us that $T$ is a maximal order, as required.

(ii) Suppose that the order in $G$ is a unit in $S$, and that $T$ is a prime maximal order. That (a) holds is immediate from Lemma 3.8. Let $p_{0}$ belong to $\Omega_{0}$. Since $p_{0}$ is reflexive, it follows that $p_{0} T$ is the annihilator in $T$ of $p_{0}^{*} T / T$, and so $p_{0} T$ is also reflexive. Therefore $p_{0} T$ is contained in a maximal reflexive prime ideal $P$ of $T$, which is a height-1 prime of $T$ by [7, Lemma 2.1] (since $T$ is a maximal order). Then, by [13, Theorem 16.6], $P \cap S=p_{0}$. It now follows from Proposition 3.12 that $P=p_{0} T$, so that $p_{0} T$ is a prime ideal of $T$. This proves (b), and so (ii) holds.

We now give an example to show that statement (ii) of the Main Theorem does not hold without the hypothesis that the order of $G$ be a unit in $S$. Note, however, that $G$ is not $X$-outer in this example. 


\subsection{ExAmPLE. Let $k=\mathbb{Z} / 2 \mathbb{Z}$ and put}

$$
S=k\left[x, x^{-1}\right][y, d / d x],
$$

the differential operator ring. Let $G=\langle g\rangle$ be a cyclic group of order 2 acting on $S$ via $s^{g}=x s x^{-1}$, for all $s \in S$. Now, $S$ is finitely generated as a module over its centre $k\left[x^{2}, x^{-2}, y^{2}\right]$, and so it is a prime Noetherian PI ring. Let $M=\left(x^{2}-1\right) S$, a $G$-invariant height-1 prime ideal of $S$. Put $\bar{S}=S / M$ and consider the skew group ring $\bar{S} * G$. Then

$$
(\bar{x} g-\overline{1})^{2}=\left(\bar{x}^{2} g^{2}-2 \bar{x} g+\overline{1}\right)=\bar{x}^{2} g^{2}-\overline{1}=\bar{x}^{2}-\overline{1}=0,
$$

since $k$ has characteristic 2 and $G$ has order 2 . Hence $(\bar{x} g-\overline{1})$ is a non-zero central nilpotent element of $\bar{S} * G$, and so

$$
(S * G) /(M * G)=(S / M) * G=\bar{S} * G
$$

is not semisimple Artinian. Therefore $M * G$ is not a semiprime ideal of $T=S * G$, and so condition (b) of Theorem 3.13 does not hold. But $T$ is a maximal order, as can be seen using the Test Theorem (Theorem 3.2). In more detail, it can be checked that all height-1 prime ideals of $T$ are localisable; hence we deduce (i) and (iv) of Theorem 3.2; since $T$ is FBN, (iii) of Theorem 3.2 is vacuous. Finally, part (ii) of Theorem 3.2 follows from [17, Example 6.2] and Proposition 2.7. Hence this example shows that $T$, being a prime maximal order, does not imply condition (b) of Theoem 3.13 when the order of $G$ is not a unit in $S$.

\section{Commutative Coefficients.}

4.1 In this section, we prove a result (Theroem 4.6) analogous to Theorem 3.13 for the case where the coefficient ring is commutative. The following result is an easy generalisation of [14, Theroem 10.1.16], where the case with $R$ a field is given. Our definition of local is given in 2.6.

4.2 TheOREM. Let $R$ be a ring and $G$ a finite group. Then the group ring $R G$ is local if and only if the following conditions hold:

(i) $R$ is local;

(ii) when $\operatorname{char}(R / J(R))=0, G=\{1\}$; and when $\operatorname{char}(R / J(R))=\rho>0$, G is a $\rho$-group.

4.3 Lemma. Let $S$ be a ring and $G$ a finite group acting on $S$. Denote the skew group ring $S * G$ by $T$, and suppose that $T$ is local. Let $p$ be a maximal ideal of $S$. Define

$$
H(p):=\left\{g \in G: p^{g}=p ; g \text { acts as identity on } S / p\right\} \text {. }
$$

Then $H(p)=\{1\}$ if $\operatorname{char}(S / p)=0$, and $H(p)$ is a $\rho$-group if $\operatorname{char}(S / p)=\rho>0$.

Proof. Suppose that $T$ is local, but that $H:=H(p)$ does not satisfy the hypotheses of the lemma. Put

$$
R:=(S / p) * H=(S / p) H,
$$

the ordinary group ring. We claim that $R$ is not local.

Consider when $\operatorname{char}(S / p)=0$; then $H \neq\{1\}$. By Maschke's Theorem $R$ is semisimple Artinian, but $R$ is not simple since $H \neq\{1\}$. Therefore $R$ is not local. In the case where $\operatorname{char}(S / p)=\rho>0, H$ is not a $\rho$-group and so $R$ is not local by Theorem 4.2. This proves the claim. Since $T / J(T)$ is Artinian, it is easy to see that $S / p$, and hence $R$, are both Artinian. 
In the following, $\operatorname{char}(S / p)$ may be either 0 or $\rho$. Since $H$ is normal in $K:=K(p)=$ $\left\{g \in G: p^{g}=p\right\}$,

$$
(S / p) * K=(S / p) H *(K / H)=R *(K / H) .
$$

Consider the augmentation ideal $A$ of $R$. Since $R$ is, not local, there exists a prime ideal $B$ of $R$ different from $A$. If a non-identity element $k$ of $K$ is such that $B^{k}=A$, then $B=\left(B^{k}\right)^{l}=A^{l}=A$ (where $l=k^{-1}$ ) since $A$ is $K$-invariant, a contradiction. Hence no $K$-conjugate of $B$ can equal $A$, and so there are distinct $K$-orbits $O_{1}, O_{2}$ of primes of $R$. Put

$$
N_{1}=\bigcap\left\{P: P \in O_{1}\right\} \quad \text { and } \quad N_{2}=\bigcap\left\{Q: Q \in O_{2}\right\} .
$$

By Lying Over (LO) ([13, Theorem 16.6]) there exist maximal ideals $M_{1}, M_{2}$ of $R *(K / H)$ with $M_{1} \cap R=N_{1}$ and $M_{2} \cap R=N_{2}$. If $M_{1}=M_{2}$, then $N_{1}=N_{2}$, a contradiction to the fact that $O_{1} \neq O_{2}$. Hence $M_{1} \neq M_{2}$ and we have distinct maximal ideals of $(S / p) * K$. Put $p_{0}=\bigcap\left\{p^{g}: g \in G\right\}$ and let $\bar{M}_{1}, \bar{M}_{2}$ be the inverse images in $\left(S / p_{0}\right) * K$ of $M_{1}, M_{2}$ respectively. Note that $\bar{M}_{1} \neq \bar{M}_{2}$, but

$$
p / p_{0}=\bar{M}_{1} \cap\left(S / p_{0}\right)=\bar{M}_{2} \cap\left(S / p_{0}\right) .
$$

Using [13, Theorem 14.7] we see that there exist distinct maximal ideals of $T$, a contradiction to the fact that $T$ is local. Hence the result holds.

4.4 Notation and Definition. Let $S$ be a commutative Noetherian domain and $G$ a finite group acting on $S$. Let $T$ denote the skew group ring $S * G$. For each $1 \neq g \in G$, define

$$
I(g)=\left\{s-s^{g}: s \in S\right\} S .
$$

It is clear that each $I(g)$ is an ideal of $S$. It is easy to check that, for any ideal $J$ of $S$, the subset $\{g \in G: I(g) \subseteq J\}$ is a subgroup of $G$; in fact it is the largest subgroup of $G$ which fixes $J$ as a set and acts trivially on $S / J$.

If we impose the condition that each non-identity element of $G$ acts non-trivially on $S$, then this ensures that $T$ is prime.

4.5 Lemma. Let $S, G$ and $T$ be as in 4.4. Then $T$ is a prime ring if and only if there does not exist a non-identity element of $G$ which acts trivially on $S$.

Proof. First suppose that $T$ is prime. Let

$$
H=\{g \in G: g \text { acts trivially on } S\}
$$

and suppose that $H \neq\{1\}$. Note first that $H$ is a normal subgroup of $G$, being the kernel of the map from $G$ into $\operatorname{Aut}(S)$. Put $\hat{H}=\sum\{h: h \in H\}$; it is clear that $\hat{H} \neq 0$. Note that $\hat{H}$ commutes with all $g \in G$. Also, $\hat{H}$ acts trivially on elements of $S$, by definition, so that $\hat{H}$ is central in $T$. Choose $1 \neq h \in H$. Then, for all $t \in T, 0=t \hat{H}(h-1)=\hat{H} t(h-1)$ so that $T \hat{H} T(h-1) T=0$; a contradiction to the primeness of $T$. Therefore $H=\{1\}$, and no non-identity element of $G$ acts trivially on $S$.

On the other hand, assume that no non-identity element of $G$ acts trivially on $S$. As in the proof of Lemma 3.5, $T$ has quotient ring $Q(T)=Q(S) * G$. By hypothesis, $G$ is a 
group of $X$-outer automorphisms of $S$, and hence of $Q(S)$. Therefore the set of $X$-inner automorphisms, $G_{\mathrm{inn}}$ of $Q(S)$ is equal to $\{1\}$. Now $Q(S)$ is a field and so is certainly prime. Using [13, Corollary 12.6] $Q(S) * G$ is also prime. Therefore $Q(T)=Q(S) * G$ is simple Artinian and so $T$ is prime, as required.

We now turn to the main result of this section, the proof of which appears as 4.12.

4.6 THEOREM. Let $S, G$ and $T$ be as in 4.4. Then $T$ is a prime maximal order if and only if the following conditions hold:

(a) $S$ is integrally closed;

(b) there exists no non-identity element $g$ of $G$ such that $I(g) \subseteq p$, for some height-1 prime $p$ of $S$.

For the case where the coefficient ring is a Dedekind domain, the corresponding result appears in [15, Theorem 40.14], and is due to Auslander, Goldman and Rim.

Remark. Recall from [8, Proposition 5.1.3] that a commutative Noetherian domain is a maximal order precisely when it is integrally closed; Lemma 2.3 then implies the equivalence of condition (a) above and condition (a) of Theorem 3.13. The following result together with Remark 3.6 shows how condition (b) above implies condition (b) of Theorem 3.13.

Observe that Proposition 4.7 cannot be generalised to non-commutative coefficient rings $S$; to see this, take $T=S * G$ as in Example 3.14 and $P$ to be a prime ideal of $T$ minimal over $M * G$. (In fact, there is only one such $P$.) It is easy to calculate that, in this example, $y-y^{g}=x^{-1} \in I(g)$, so that $I(g)=S$.

4.7 Proposition. Adopt the notation of 4.4 and suppose there exist no non-identity elements of $G$ acting trivially on $S$. Let $P \in \operatorname{Spec}(T)$. Put

$$
\Psi:=\{p \in \operatorname{Spec}(S): p \text { is minimal over } P \cap S\} .
$$

Suppose further that $I(g) \nsubseteq p$, for all $1 \neq g \in G, p \in \Psi$. Then $P=(P \cap S) T$.

Proof. We know that $(P \cap S) T \subseteq P$ and that by [13, Lemma 14.1] $P \cap S$ is a $G$-prime ideal of $S$. Now, $P$ lies over $P \cap S$ and using [13, Theorem 16.6], Incomparability implies that there does not exist $P_{1} \in \operatorname{Spec}(T)$ with $P_{1} \subset P$ and $P_{1} \cap S=P \cap S$. Therefore $P$ is minimal over $(P \cap S) T$. To show that $(P \cap S) T=P$ it is enough to show that $(P \cap S) T \in \operatorname{Spec}(T)$. Consider

$$
T /((P \cap S) T) \cong(S /(P \cap S)) * G
$$

we show that the latter ring is prime. It is clear that $S /(P \cap S)$ is $G$-prime. Let $p \in \Psi$. Then $p /(P \cap S)$ is a minimal prime of $S /(P \cap S)$. Put $K:=\left\{g \in G: p^{g}=p\right\}$. Since $I(g) \nsubseteq p$ for all $1 \neq g \in G$, no element of $K$ acts trivially on $S / p$. Lemma 4.5 then gives that $(S / p) * K$ is prime. Now,

$$
\{(S /(P \cap S)) /(p /(P \cap S))\} * K \cong(S / p) * K
$$

and so by [13, Corollary 14.8$],(S /(P \cap S)) * G$ is prime. Therefore $T /((P \cap S) T)$ is prime, $(P \cap S) T \in \operatorname{Spec}(T)$ and $P=(P \cap S) T$, as required.

4.8 Proposition. Let $S, G$, and $T$ be as in 4.4 , and let $P$ be a prime ideal of $T$. If $P=(P \cap S) T$, then $P$ is localisable. 
Proof. By [8, Theorem 4.2.7], all ideals of $S$ have the right $A R$-property since $S$ is a commutative Noetherian domain. In particular, $P \cap S$ does. Then, by [17, Lemma 4.4], $P=(P \cap S) T$ has the right and left $A R$-property. Using [8, Proposition 6.8.21(ii)], we see that $P$ is localisable.

In view of Proposition 4.7, it follows immediately from Theorem 3.13 that conditions (a) and (b) of Theorem 4.6 are sufficient for $T$ to be a maximal order. We now concentrate on the necessity of these conditions.

4.9 Lemma. Adopt the notation of 4.4 and suppose that each non-identity element of $G$ acts non-trivially on $S$. Let $P$ be a localisable height-1 prime of $T$ and put $p_{0}=P \cap S$. Let $q:=P \cap Z=p_{0} \cap Z$, a height-1 prime of $Z$, where $Z$ is the centre of $T$. Then $T_{P}=T_{p_{0}}=T_{q}$. In particular, $T_{p_{0}}$ is a local ring.

Proof. Since $T$ is a maximal order, by hypothesis, $P$ is localisable by Theorem 3.2. By [5, Lemma 12.17], the clique of $P$ is equal to $\{P\}$. Thus, by [5, Theorem 11.20] (also discussed in [11]), $P$ is the one and only prime ideal of $T$ lying over $P \cap Z=q$. The proof is now routine.

4.10 Proposition. Adopt the notation of 4.4 and suppose that each non-identity element of $G$ acts non-trivially on $S$. Let $P$ be a localisable height-1 prime of $T$, and put $p_{0}=P \cap S=\bigcap\left\{p^{g}: g \in G\right\}$ for some height-1 prime $p$ of $S$ (see Lemma 3.5). Suppose that $\operatorname{char}(S / p)=0$. Then there exists no non-identity element $g$ of. $G$ such that $I(g) \subseteq p$.

Proof. Since $T_{p_{0}}$ is local, by Lemma 4.9, Lemma 4.3 implies that $H(p)=\{1\}$ (where $H(p)$ is as defined in Lemma 4.3). It is now immediate that there exists no non-identity element $g \in G$ such that $I(g) \subseteq p$, as required.

We now prove an analogous result to Proposition 4.10 for the case where $S / p$ has positive characteristic.

4.11 Proposition. Adopt the notation of 4.4 and suppose that each non-identity element of $G$ acts non-trivially on $S$. Assume that $T$ is a maximal order. Let $p$ be a height -1 prime of $S$, and suppose that $\operatorname{char}(S / p)=\rho>0$. Then no non-identity element $g$ of $G$ is such that $I(g) \subseteq p$.

Proof. Firstly, $T_{p_{0}}$ is a local ring by Lemma 4.9. Let $H=H(p)$. By Lemma $4.3, H$ is a $\rho$-group. Let $1 \neq x \in H$ be such that $x$ has order $\rho$. First we claim that $T_{p_{0}}$ has infinite global dimension. For, put $N:=p S_{p_{0}}$, a maximal ideal of $S_{p_{0}}$. Note that $S_{p_{0}} / N \cong Q(S / p)$, and that $\operatorname{char}\left(S_{p_{0}} / N\right)=\rho$. Define

$$
G(N):=\left\{g \in G: s-s^{g} \in N \text { for all } s \in S_{p_{0}}\right\} .
$$

We have $x \in G(N)$ and $x$ has order $\rho$. Therefore, by [17, Theorem 5.2] gl.dim. $\left(T_{p_{0}}\right)=\infty$, as claimed.

Now, since $T_{p_{0}}$ has a unique non-zero prime ideal $J:=J\left(T_{p_{0}}\right)$, it is clear that $J$ is reflexive. Then Proposition 2.7 gives that $T_{p_{0}}$ is not a maximal order. It follows that $T$ is not a maximal order, a contradiction to our initial hypothesis. Therefore there exists no such element $x$, and so $H=\{1\}$. This completes the proof. 
4.12 Proof of Theorem 4.6. As was noted above, the sufficiency of conditions (a) and (b) of Theorem 4.6 is a direct consequence of Theorem 3.13. For the converse, suppose that $T$ is a prime maximal order. That (a) holds follows from Lemma 3.8 together with Lemma 2.3 and the fact that a commutative Noetherian domain is a maximal order precisely when it is integrally closed. Condition (b) is then proved necessary by Proposition 4.10 and Proposition 4.11.

\section{REFERENCES}

1. M. Chamarie, Anneaux de Krull non commutatifs, J. Algebra 72 (1981), 210-222.

2. J. H. Cozzens and F. L. Sandomierski, Maximal orders and localisation I, J. Algebra 44 (1987), 319-338. 86-104.

3. D. Eisenbud and J. C. Robson, Hereditary Noetherian prime rings, J. Algebra 16 (1970),

4. K. R. Fuller and W. A. Shutters, Projective modules over non-commutative semilocal rings, Tôhoku Math. J. (2) 27 (1975), 303-311.

5. K. R. Goodearl and R. B. Warfield, Jr., An introduction to noncommutative Noetherian rings, L.M.S. Student Texts 16 (C.U.P. 1989). 441-449.

6. C. R. Hajarnavis and T. H. Lenagan, Localisation in Asano orders, J. Algebra 21 (1972), 375-384 1987).

7. C. R. Hajarnavis and S. Williams, Maximal orders in Artinian rings, J. Algebra 90 (1984), (1993).

8. J. C. McConnell and J. C. Robson, Noncommutative Noetherian rings (Wiley-Interscience,

9. R. Martin, Skew group rings and maximal orders, Ph.D. Thesis, University of Glasgow

10. G. Maury and J. Raynaud, Ordres maximaux au sens de K. Asano (Springer-Verlag, 1980).

11. B. J. Müller, Localisation in fully bounded Noetherian rings, Pacific J. Math. 67 (1976), 233-245.

12. E. Nauwelaerts and F. van Oystaeyen, Finitely generalised crossed products over tame and maximal orders, J. Algebra 101 (1986), 61-68.

13. D. S. Passman, Infinite crossed products (Academic Press, 1989).

14. D. S. Passman, The algebraic structure of group rings (Wiley-Interscience, 1977).

15. I. Reiner, Maximal orders (Academic Press, 1975).

16. P. F. Smith, Quotient rings of group rings, J. London Math. Soc. (2) 3 (1971), 645-660.

17. $\mathrm{Z}$. Yi, Homological dimension of skew group rings and crossed products, J. Algebra 164 (1994), 101-123.

Department of Mathematics

UNIVERSITY OF GLASGOW

UNIVERSITY GARDENS

Glasgow G12 8QW 